\title{
Dam Instability Risk Analysis of Damaged Spillway
}

\author{
Gholamhossein Akbari \\ Civil Engineering, University of Sistan and Baluchestan, Zahedan, Iran \\ E-mail: gakbari@hamoon.usb.ac.ir
}

\begin{abstract}
Parametric errors were analyzed for MASHKID SOFLA Dam Reservoir System. Errors in cavitation number examined for a complex system of Spillways operation and found a potential risk to hydraulic structural stability. The necessary measures for cavitation number, the ways to analyze parametric errors induced through governing equations were investigated. The calculation methods for cavitation number were found liable to risk. A computer coded MATLAB program was established for the selected case study and spillway dam stability problem was investigated. During spilling of flooding water over surface area deteriorations of concrete was observed because of cavitations and the collision of flowing water. Cavitation phenomenon was the cause for holes generation and concerns for severe damages to ogee spillway - dam instability.
\end{abstract}

Keywords: Cavitation index, spillway system damage, dam instability, risk analysis modeling, failure zone.

ENGINEERING JOURNAL Volume 17 Issue 4

Received 20 September 2012

Accepted 11 March 2013

Published 1 October 2013

Online at http://www.engj.org/

DOI:10.4186/ej.2013.17.4.1 


\section{Introduction}

Dam construction in arid zone of the world plays a noticeable role in providing drinking water, irrigation and industrial development. Recent years, poor rain falls have been highly varied dependent on both temporal and spatial changes [1]. As the result of global warming, flooding in rivers over flown from reservoirs, causes considerable risk to dam stability. Most of this flood water should be transferred from the spillway structure to the lower part of dam. On the other hand, transferring storming flood waters require safe design of reservoir ogee spillway systems and stability of dams are in correlations with reliable operations of spillway. Thus, to assure suitable operation, search for solution and prevention of problems are inevitable during the exploitation. One of the most relevant problems during spilling of flooding water is surface deterioration of concrete because of cavitations and deepening during the collision of flowing water $[2,3]$. Cavitations have the main reason for holes generation and cause of severe damages the system.

Statistical performed research analyzed the change in tensile behavior of concrete varies due to explosion of cavitations. Cloud in spillways increased the explosion rate of cavitations and consequently increased number of loadings, the nonlinear behavior of concrete [4]. In this way, cavitation number and its index in spillways design is very important. Cavitation number is the important quantity of cavitation flow which indicates the flow regime. Cavitation index is a parameter increased the probability of damage as a result of cavitations

Stinebring [5] observed that the cavitation index decreased in relation to the incipient index and damage rate increased slowly. If the cavitation index is decreased further, a zone is reached where the damage rate is inversely proportional to the cavitation index. Further decrease of the index results in a point being reached where the damage rate had a maximum value. As the index is decreased even further, the damage rate decreased. From this, it appears that the cavitation intensity increased and then decreased as the cavitation index is lowered below the value of the incipient cavitation index. The noise spectrum has a similar behaviour.

Colgate [6] proposed a similar shaped curve for the damage intensity as the cavitation index decreased. In addition, he showed the area of damage continues to grow larger as the cavitation index decreased. Colgate's observations were justified in this study for the following reasons. As damage rate increased values of the cavitation index decreased and the length of the cavitation cloud increased. However, length of the cavitation cloud was found sensitive to variations in velocity. Therefore, length of the damage area enlarged as the difference between the incipient cavitation index and the cavitation index of the flow increased. The intensity, measured in pits per square centimeter per second, decreased while the total amount of material removed actually increased.

\section{Objectives}

The purpose of this research was to present application of latest scientific review and to provide the designer of hydraulic structures both an understanding of cavitations and the design tools necessary to eliminate or reduce the damaging effects of cavitations in hydraulic chute and spillway structures. In this study cavitation theory was highlighted. Cavitation characteristics of typical surface irregularities were considered. The study is applicable to many similar water drop structure systems. A discussion of cavitation damage highlights warnings to be aware of Dam instability risk endangers project. Investigations treated the influence of geometry. Analysis carried out here addresses aeration and aerators required to prevent cavitations for safety of the Dam structure. Study contains recommendations for designers to implement continuous situ investigations. This research should help in specifying surface tolerances and deciding when special designs such as aerators and supplementary safety requirements are necessary. The study concludes many recent selected literature survey $[1,7,8,9]$, numerical analysis and field applications and experiences.

\section{Methodology (Formulations and Analysis)}

Governing equations used in formulations of the problem are the continuity and the general momentum equations for two-dimensional flow in the $\boldsymbol{s}$ and $\boldsymbol{n}$ directions [7] given as:

$$
U_{S} \frac{\partial U_{S}}{\partial s}+U_{n} \frac{\partial U_{S}}{\partial n}-U_{s} U_{n} K_{s}-U_{n}^{2} K_{n}=-\frac{1}{\rho} \quad \frac{\partial P}{\partial s}-\frac{\partial \Omega}{\partial s}
$$




$$
\begin{gathered}
U_{S} \frac{\partial U_{n}}{\partial s}+U_{n} \frac{\partial U_{n}}{\partial n}-U_{s} U_{n} K_{n}-U_{s}^{2} K_{s}=-\frac{1}{\rho} \quad \frac{\partial P}{\partial n}-\frac{\partial \Omega}{\partial n} \\
K_{n}=\frac{d^{2} s}{d n^{2}}\left[1+\left(\frac{d s}{d n}\right)^{2}\right]^{-3 / 2} ; K_{s}=\frac{d^{2} n}{d s^{2}}\left[1+\left(\frac{d n}{d s}\right)^{2}\right]^{-3 / 2}
\end{gathered}
$$

where $K_{s}$ is curvature of the curve normal to streamline; $P$ is pressure intensity; $U_{n}$ is the velocity tangent to streamline; $U_{n}$ is the velocity normal to streamline; $\rho$ is density of water; $\Omega$ is potential of external force; $n$ is coordinate distance normal to streamline; and $s$ is coordinate distance tangent to streamline. However, by definition, flow does not cross a streamline. Thus, $U_{n}$ is 0 along the streamline. The potential of the external force, $\Omega$, in this case is gravity, $(\Omega=g Z) ; g$ is gravitational constant; and $Z$ is elevation. With these considerations, the equation of motion can be rewritten in the following forms:

$$
\begin{gathered}
U_{s} \frac{\partial U s}{\partial s}+\frac{1}{\rho} \quad \frac{\partial P}{\partial s}+g \frac{\partial Z}{\partial s}=0 \\
\frac{\partial}{\partial s}\left(\frac{U_{s}^{2}}{2}+\frac{P}{\rho}+g Z\right)=0 \\
\frac{\partial}{\partial n}\left(\frac{U_{S}^{2}}{2}+\frac{P}{\rho}+g Z\right)=U_{S}\left(U_{S} K_{S}-\frac{\partial U_{S}}{\partial n}\right)=0 \\
\frac{\partial}{\partial n}\left(\frac{U_{S}^{2}}{2}+\frac{P}{\rho}+g Z\right)+U_{S} \zeta=0
\end{gathered}
$$

These last three equations are significant because they show that the Bernoulli equation is constant along a streamline [8], the constant changes from streamline to stream-line as the vortices changes. In rotational flow, the vortex $(\zeta)$ is equal to twice the angular velocity $(\omega), \zeta=2 \omega$. In rotational flow, the vortex 4 is equal to zero, $\zeta=0$.

Integrating governing equations along the free water surface between the reservoir and cavitation point as shown on Fig. 2, results in

$$
\frac{U_{a}^{2}}{2 g}+Z_{a}-Z_{r}=0
$$

where $U_{a}$ is velocity at point $a, Z_{a}$ is elevation at point $a, Z_{r}$ is elevation of reservoir, the relationship between the free water surface and the point is given by

$$
Z_{a}-Z_{r}=-H_{b}+d \cos \theta
$$

where $H_{b}=$ reservoir elevation minus elevation at point $b$; $d$ equals flow depth normal to invert; and $\theta$ is angle between tangent to invert an horizontal. Thus,

$$
U_{a}=\left[2 g\left(H_{b}-d \cos \theta\right)\right]^{1 / 2}
$$

This process can be simplified by making assumptions concerning the flow vortices [9].

To apply the preceding equations to spillway chute design, the intrinsic coordinates need to be defined in terms of the Cartesian coordinate system. The slope of the tangent to the invert is given by

$$
\frac{d H_{b}}{d x}=\tan \theta=\frac{\left(1-\cos ^{2} \theta\right)^{1 / 2}}{\cos \theta}
$$

A relationship for the radius of curvature of the invert can be obtained by realizing that

$$
\frac{d \cos \theta}{d H_{b}}=-\sin \theta \quad \frac{d \theta}{d H_{b}}
$$

The cavitation index is defined as

$$
\sigma=\frac{P_{a}+P_{b}-P_{v}}{\rho U_{b}^{2} / 2}
$$




\section{Hydraulics Data and Case Study Results}

The hydraulics data for this case study was extracted from water surface profile estimation as shown in Table 1. Spillway discharges changed appreciably with a variable starting water surface elevation. Estimate the expected annual probability for initiation of cavitation damage. Assumption that the flows identified in the table are maintained for an extended period of time. Results of investigations in Fig. 1, provided that the pressure of flowing discharge $(\mathrm{Q}=155)$ was decreased through increases in the flow rate velocity, a critical condition was reached when cavitations just begin. Similarly, cavitations existed and the flow velocity was decreased and the pressure of flowing discharge $(\mathrm{Q}=200)$ was increased, a critical condition was reached when the cavitations decreased. This condition was the design cavitations as illustrated and recommended in Fig. 1. Incipient cavitations and design cavitations did not occur at the same flow conditions. The distinction was especially important in this investigation and it may be the case for many practical purposes in many hydraulic structures design. A set of critical flow conditions was considered for which the individual cavitations suddenly changed into one large void. Conditions under which the large voids occurred enveloped cavitations. This highlighted result can be seen from provided illustrations in the Fig. 3.

Comprehensive investigations were made to determine the errors in cavitation number for a damaged hydraulic structure (reservoir-dam-spillways system) Deliberating the necessary parameters for cavitation number, the way to extract them and dominant equations was made. The calculation method of cavitation number was checked against possible errors. These parameters were tested for MASHKID SOFLA Dam through a developed model and computer coded MATLAB programs. A selected case problem was solved for a spillway system failure.

Despite the spillway surface was 'smooth' and well-constructed. Values of absolute roughness of $1 \mathrm{~mm}$ for $5.5 \mathrm{~m}$ lengths and widths were the smoothest surface was considered.

The causes for concerns were the errors in spillways and dam establishment provided cavitation problems at velocities exceeding $35 \mathrm{~m} / \mathrm{s}$.

Dissolved gases, particles in suspensions were also involved in the cavitation process at higher pressures. Errors in pressures below $7 \mathrm{~m}$ vacuum ( $3 \mathrm{~m}$ absolute) in design were investigated. It was found that turbulent flows and the mean pressure were well above the danger limits. Cavitations occurred owing to fluctuating instantaneous pressures that fell below the limit. The danger of cavitations was assessed to analyze the turbulent pressure fluctuations. The danger of cavitations was ascertained through the model and prototype measurements were provided, the errors in cavitation number for design was highlighted. Danger of cavitation damage; Errors in the design or the mode of operations of the complicated huge hydraulic structure was found effective for damage analysis.

Table 1. Flow velocities and cavitation indices at spillway station.

\begin{tabular}{cccc}
\hline $\begin{array}{c}\text { Flood Frequnecy, } \\
\text { yrs }\end{array}$ & $\begin{array}{c}\text { Spillway Discharge, } \\
\mathrm{m}^{3} / \mathrm{s}^{*}\end{array}$ & $\begin{array}{c}\text { Flow Velocity, } \\
\mathrm{m} / \mathrm{s}\end{array}$ & Cavitation Index \\
\hline 100 & 2000 & 40 & 1.03 \\
500 & 7300 & 55 & 0.50 \\
1000 & 17800 & 88 & 0.31 \\
10000 & 25300 & 91 & 0.20 \\
\hline
\end{tabular}




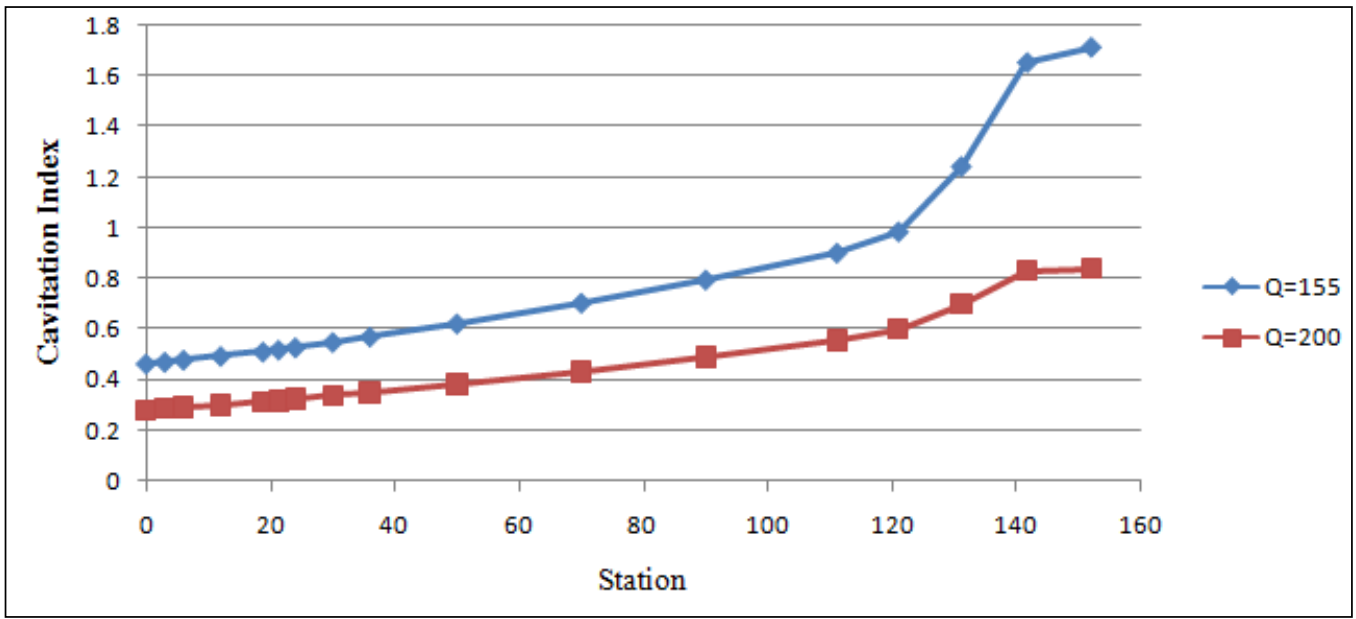

Fig. 1. Cavitation index at downstream dam-spillways system.

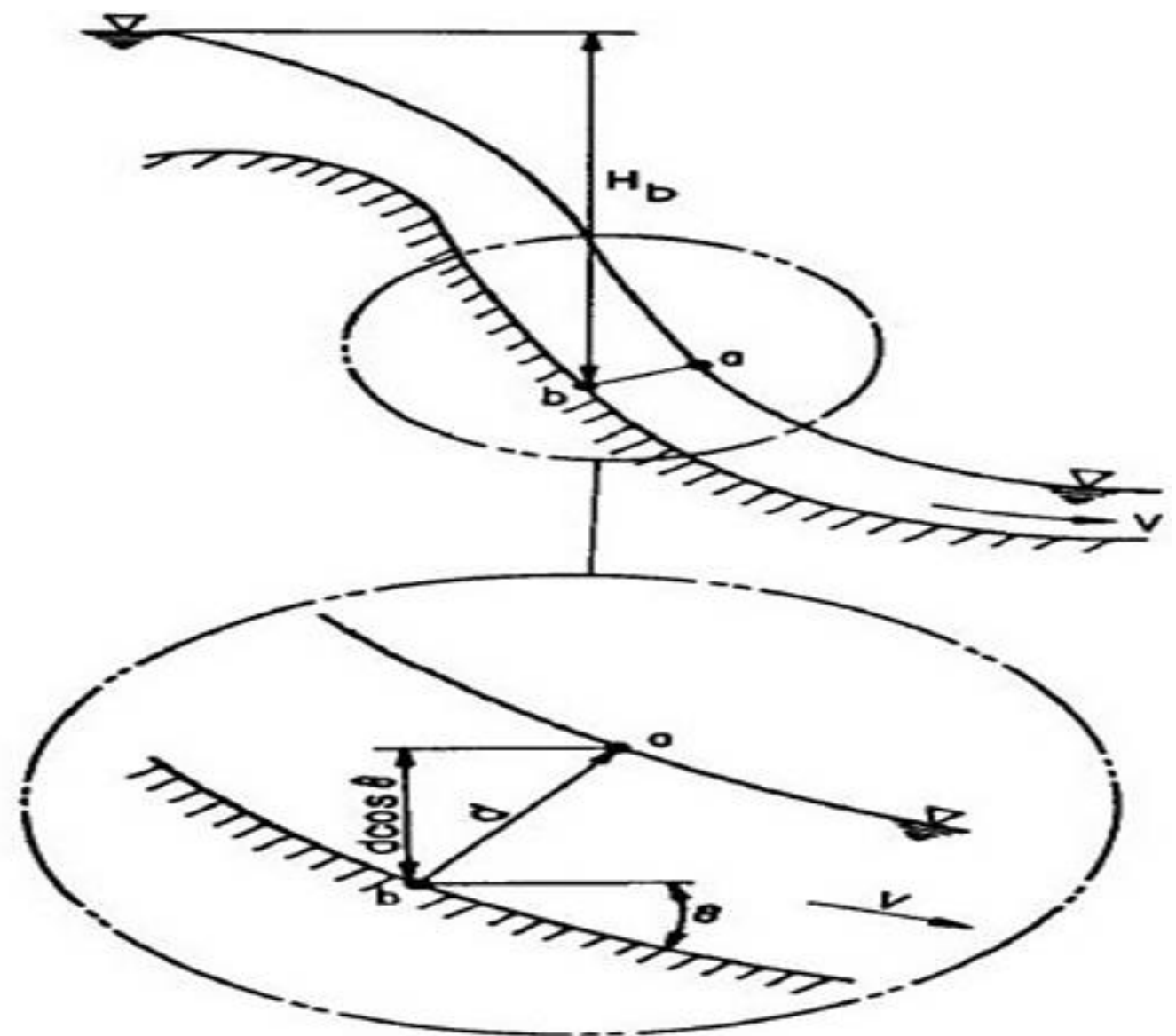

Fig. 2. Geometry and schematic presentation of spillways $\mathrm{D} / \mathrm{S}$ of dam. 

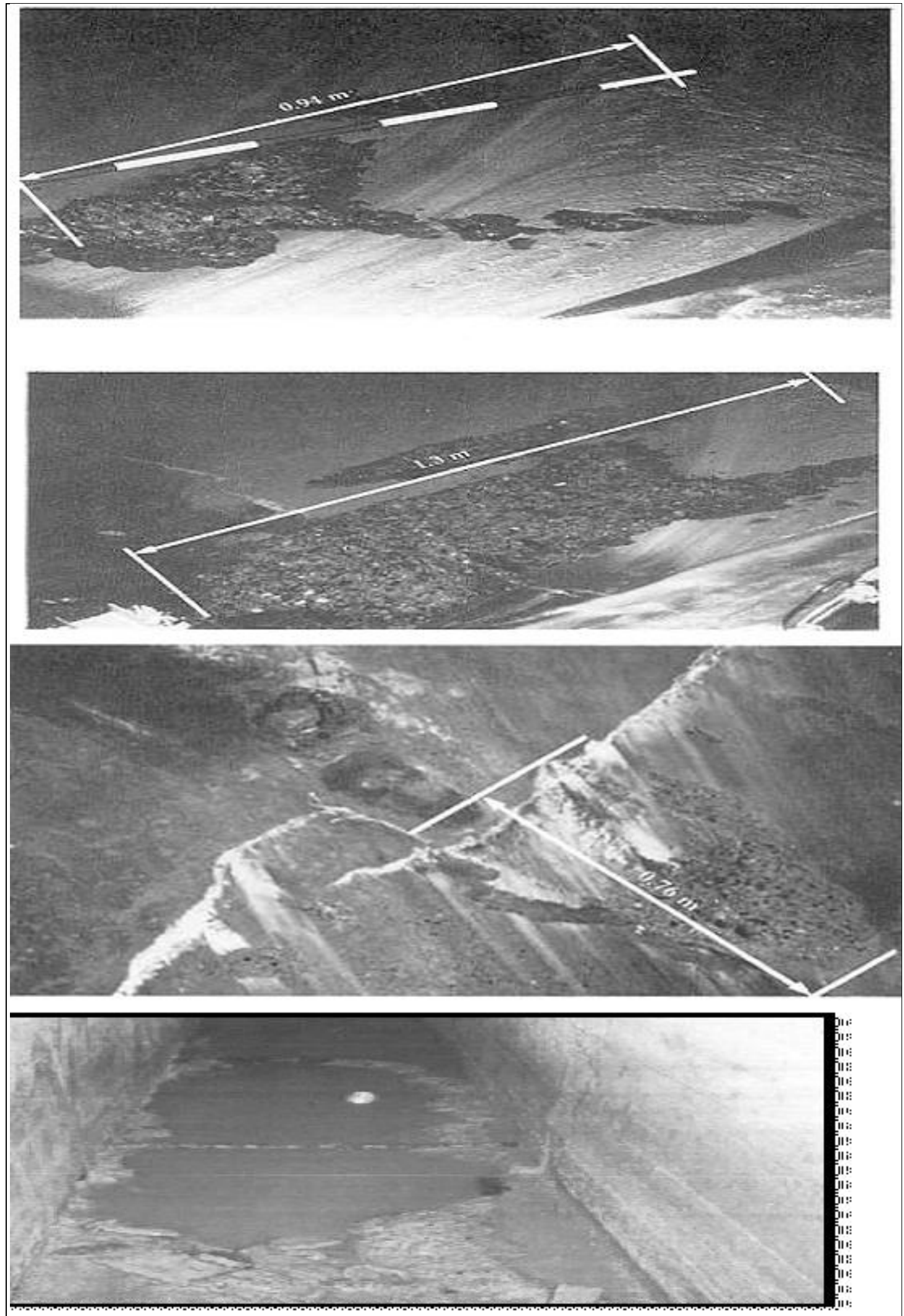

Fig. 3. Cavitation observations at downstream dam-spillways system. 


\section{Discussions}

Cavitations occurred when the pressure in the flow of water dropped to the values of the pressure of the saturated water vapor, cavities filled by vapor, as a result of the low pressures were formed. Low pressureswell below atmospheric pressure occurred at points of separation of water flowing over spillways, particularly the flow velocity was high.

After cavitations, the cavitation number felt below a critical value which was a function of the geometry for the case studied (Fig. 2). The cavitation index of the flow was determined on the order of one-sixth of the incipient cavitation index, an irregularity at distances up to100 times the height of the irregularity was considered. In Fig. 3, damage in hydraulic structure was in the decreasing range of damage rates. Rarely are laboratory experiments made in this range because of the difficulty to create a facility that was produce damage at such low values of the cavitation index.

Two factors, pressure and velocity influenced the onset of cavitations. They were combined with density in the cavitation number.

Introduction of air at the endangered parts (artificial aeration), not prevented the occurrence of extremely low pressures were required, these errors were introduced as sources of damages for establishments. The use of special materials was not provided and substantially not postponed the onset of cavitation damage.

\section{Acknowledgments}

Cavitations in damaged Spillways have been studied for over years by USBR. To acknowledge all who contributed in this field is impossible. Nevertheless, some contributors deserve special recognition. Many thanks to H. T. Falvey, A. F. Babb, R. E. A. Arndt, F. DeFazio, T. J. Rhone. Appreciation is expressed to my Post -grad students Ellias Vahdat, Liegh Rafaat, Naderian, Jaafarpour for their group working under my research team.

\section{References}

[1] G. H. Akbari, A. R. Hosseinnezhad, and R. Barati, "Developing a model for analysis of uncertainties in prediction of floods," Journal of Advanced Research, vol. 3, pp. 73-79, 2012.

[2] R. E. A. Arndt, "Recent advances in cavitation research," in Advances in Hydroscience, vol. 12, V. T. Chou, Ed., New York: Academic Press, 1981.

[3] ASCE, Hydraulic Design of Spillways: Technical engineering and design guides adapted from US Army Corps of Engineers, no. 12, New York: ASCE, 1995.

[4] H. Chanson, Hydraulic Design of Stepped Cascades, Channels, Weirs and Spillways, Oxford: Pergamon, 1995.

[5] D. A. Ervine and G. S. C. Oliver, "The full scale behaviour of air regulated siphon spillways," in Proceedings of the Institution of Civil Engineers, Part 2, 1980, vol. 69, pp. 687-706.

[6] H. T. Falvey, "Cavitation in chutes and spillways," Water Resources Technical Publication: Engineering Monograph 442, Denver: Bureau of Reclamation, 1990.

[7] N. L. Pinto, "Prototype aerator measurement," in Air Entrainment in Free Surface Flows: LAHR Hydraulic Structures Design Manual, vol. 4, I. R. Wood, Ed., Rotterdam: Balkema, 1991.

[8] D. L. Vischer and W. H. Hager, Dam Hydraulics, Chichester: John Wiley \& Sons, 1998.

[9] I. R. Wood, "Free surface air entrainment on spillways," in Air Entrainment in Free Surface Flows: LAHR Hydraulic Structures Design Manual, vol. 4, I. R. Wood, Ed., Rotterdam: Balkema, 1991. 
